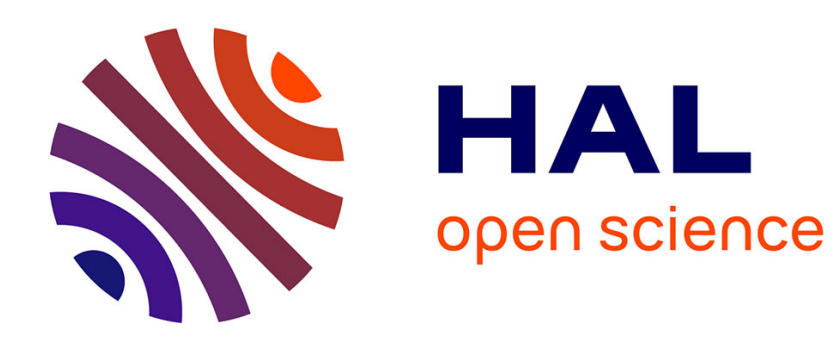

\title{
TWO GENERALISATIONS OF THE TITCHMARSH DIVISOR PROBLEM
}

\author{
Jie $\mathrm{Wu}$
}

\section{To cite this version:}

Jie Wu. TWO GENERALISATIONS OF THE TITCHMARSH DIVISOR PROBLEM. 2019. hal02322940

\section{HAL Id: hal-02322940 \\ https://hal.science/hal-02322940}

Preprint submitted on 21 Oct 2019

HAL is a multi-disciplinary open access archive for the deposit and dissemination of scientific research documents, whether they are published or not. The documents may come from teaching and research institutions in France or abroad, or from public or private research centers.
L'archive ouverte pluridisciplinaire HAL, est destinée au dépôt et à la diffusion de documents scientifiques de niveau recherche, publiés ou non, émanant des établissements d'enseignement et de recherche français ou étrangers, des laboratoires publics ou privés. 


\title{
TWO GENERALISATIONS OF THE TITCHMARSH DIVISOR PROBLEM
}

\author{
JIE WU
}

\begin{abstract}
In this paper, we considered two generalisations of the classical Titchmarsh divisor problem: friable variant and short intervals.
\end{abstract}

\section{INTRODUCTION}

As usual, denote by $\tau(n)$ the number of divisors of the integer $n$ and by $\varphi(n)$ the Euler totient function. The letter $p$ is used to denote primes. The Titchmarsh divisor problem consists to evaluate the asymptotic behaviour of the counting function $\sum_{p \leqslant x} \tau(p-a)$ for $x \rightarrow \infty$, where $a \in \mathbb{Z}^{*}$ is a fixed non-zero integer. This problem was studied initially by Titchmarsh [15], who showed, under the Generalised Riemann Hypothesis (GRH) for the Dirichlet $L$-functions,

$$
\sum_{p \leqslant x} \tau(p-a) \sim C_{a} x
$$

for $x \rightarrow \infty$, where

$$
C_{a}:=\frac{\varphi(a)}{a} \prod_{p \nmid a}\left(1+\frac{1}{p(p-1)}\right) .
$$

Linnik [8] removed GRH by his dispersion methd. The best known result is due to Fouvry [3] and Bombieri-Friedlander-Iwaniec [1], who proved, independently, for any $A>1$,

$$
\sum_{p \leqslant x} \tau(p-a)=C_{a} x+D_{a} \int_{2}^{x} \frac{\mathrm{d} t}{\log t}+O_{A}\left(\frac{x}{(\log x)^{A}}\right),
$$

where $D_{a}$ is certain constant depending on $a$. Diverse generalisations were studied by different authors. Here we only present two such generalisations:

(i) Very recently Xi [18] considered a quadratic analogue of (1.1) and established the following inequalities

$$
\{0.5-o(1)\} \frac{6}{\pi^{2}} x \leqslant \sum_{p \leqslant x} \tau\left(p^{2}+1\right) \leqslant 3.496 \cdot \frac{6}{\pi^{2}} x
$$

for $x \rightarrow \infty$. The expected asymptotic formula is

$$
\sum_{p \leqslant x} \tau\left(p^{2}+1\right) \underset{x \rightarrow \infty}{\sim} \frac{6}{\pi^{2}} x .
$$

Date: June 11, 2018.

2010 Mathematics Subject Classification. 11N05, 11N25, 11N36.

Key words and phrases. Shifted prime, Friable integer, Sieve. 
(ii) Pollack [11] studied analogues of (1.1) for elliptic curves and proved the following two results:

- For the CM elliptic curve $E: y^{2}=x^{3}-x$, there is an explicite constant $C$ such that

$$
\sum_{\substack{p \leqslant x \\ p \equiv 1(\bmod 4)}} \tau\left(\left|E\left(\mathbb{F}_{p}\right)\right|\right) \sim C x,
$$

as $x \rightarrow \infty$, where $\left|E\left(\mathbb{F}_{p}\right)\right|$ is the order of the group of points of the reduction at $p$ of $E$.

- Let $E$ be a non-CM elliptic curve over $\mathbb{Q}$. Assume the GRH for Dedekind zetafunctions. Then for $x \rightarrow \infty$, we have

$$
\sum_{p \leqslant x}^{*} \tau\left(\left|E\left(\mathbb{F}_{p}\right)\right|\right) \asymp_{E} x
$$

where $\sum^{*}$ means that the sum is restricted to primes of good reduction and the implied constants depend on $E$.

In this paper, we shall consider two other generalisations of the classic Titchmarsh divisor problem: friable variant and short intervals.

Firstly let us fix some notation. As usual, denote by $P^{+}(n)$ the largest prime factor of integer $n$ with the convention $P^{+}(1)=1$. We say that an integer $n$ is $y$-friable if $P^{+}(n) \leqslant y$. Let $\rho(u)$ be the Dickman function, which is defined as the unique continuous solution of the differential-difference equation

$$
\begin{cases}\rho(u)=1 & (0 \leqslant u \leqslant 1), \\ u \rho^{\prime}(u)=-\rho(u-1) & (u>1) .\end{cases}
$$

For $x \geqslant 1$ and $y>1$, we use systematically the notation

$$
u:=\frac{\log x}{\log y}
$$

For $a \in \mathbb{Z}^{*}, q \in \mathbb{N}$ such that $(a, q)=1$ and $x \geqslant 1, y>1$, define

$$
\pi(x, y ; d, a):=\sum_{\substack{p \leqslant x \\ p \equiv a(\bmod d) \\ P^{+}((p-a) / d) \leqslant y}} 1,
$$

which counts the number of primes $p \leqslant x$ in the arithmetic progression with friable indices $\{a+m d\}_{m} y$-friable. A key point to study friable variant of the Titchmarsh divisor problem (1.1) is that we need a theorem of Bombieri-Vinogradov type for $\pi(x, y ; d, a)$. It seems that this is a very interesting and rather difficult question, since Pomerance's conjecture (see [12])

$$
\pi(x, y ; 1, a) \sim \pi(x) \rho(u)
$$

is still open, where $u:=(\log x) / \log y$ and $\pi(x)$ is the number of primes $\leqslant x$. Granville $[5$, Section 5.3] announced that (1.4) follows from the Elliott-Halberstam conjecture without proof. Very recently Wang [16] gave a detailed proof of such result: Let $a \in \mathbb{Z}^{*}$ be a fixed 
non-zero integer. Assuming that for any $A>0$ and $\varepsilon>0$ there is a positive number $\eta=\eta(A, \varepsilon)$ such that

$$
\sum_{d \leqslant x^{1-\eta}}\left|\sum_{\substack{p \leqslant x \\ p \equiv a(\bmod d)}} 1-\frac{\pi(x)}{\varphi(d)}\right| \ll_{a, A, \varepsilon} \frac{x}{(\log x)^{A}}
$$

then we have

$$
\sum_{\substack{p \leqslant x \\ P^{+}(p-a) \leqslant y}} 1=\pi(x) \rho(u)\left\{1+O_{a}(\varepsilon)\right\}
$$

for $x \geqslant y \geqslant x^{\varepsilon}$, where the implied constant depends on $a$ only.

Our result on the friable variant of the classic Titchmarsh divisor problem (1.1) is as follows, which is comparable with (1.5).

Theorem 1. Let $a \in \mathbb{Z}^{*}$ be a fixed non-zero integer. For any $\varepsilon>0$, there exists a positive number $\eta=\eta(\varepsilon)$ such that if we assume the Elliott-Halberstam conjecture $\mathrm{EH}[\eta]$, we have

$$
\sum_{\substack{p \leqslant x \\ P^{+}(p-a) \leqslant y}} \tau(p-a)=C_{a} \pi(x)(\log y)(\rho * \rho)(u)\left\{1+O_{a}(\varepsilon)\right\}
$$

uniformly for $x \geqslant 2$ and $x \geqslant y \geqslant x^{\varepsilon}$, where the convolution of $\rho$ and $\rho$ is defined by

$$
(\rho * \rho)(u):=\int_{0}^{u} \rho(v) \rho(u-v) \mathrm{d} v
$$

and the implied constant depends on a only.

About the generalisation of (1.1) to short intervals, we have the following result.

Theorem 2. Let $a \in \mathbb{Z}^{*}$ a fixed non-zero integer and $\frac{3}{5}<\theta \leqslant 1$. Then for $x \rightarrow \infty$ and $y=x^{\theta}$, we have

$$
C_{a}^{\dagger}(\theta) y\left\{1+O_{a, \theta}\left(\frac{\log _{2} x}{\log x}\right)\right\} \leqslant \sum_{x<p \leqslant x+y} \tau(p-a) \leqslant C_{a}^{\sharp}(\theta) y\left\{1+O_{a, \theta}\left(\frac{\log _{2} x}{\log x}\right)\right\}
$$

where $\log _{k}$ is the $k$-fold iterated logarithm,

$$
\begin{aligned}
& C_{a}^{\dagger}(\theta):=(2 \theta-1) C_{a}, \\
& C_{a}^{\sharp}(\theta):=(2 \theta-1-4 \log (2 \theta-1)) C_{a},
\end{aligned}
$$

and the implied $O$-constants depend on a and $\theta$ at most.

Since $C_{a}^{\dagger}(1)=C_{a}^{\sharp}(1)=C_{a}$, the inequalities (1.8) with $\theta=1$ become the classic asymptotic formula (1.1). The key tool for the proof of this theorem is a Bombieri-Vinogradov theorem in short intervals (see Lemma 5.1 below). 


\section{SOME PRELIMINARY LEMMAS}

Firstly we prove a preliminary lemma, which will be needed later.

Lemma 2.1. Let $a \in \mathbb{Z}^{*}$ be a fixed non-zero integer. Then for any $\varepsilon \in(0,1)$, we have

$$
\sum_{\substack{d \leqslant x,(a, d)=1 \\ P^{+}(d) \leqslant y}} \frac{1}{\varphi(d)}=C_{a} \pi(x)(\log y) \int_{0}^{u} \rho(v) \mathrm{d} v\left\{1+O_{a, \varepsilon}\left(\frac{\log (u+1)}{\log y}\right)\right\}
$$

uniformly for

$$
x \geqslant 3 \quad \text { and } \quad \exp \left\{\left(\log _{2} x\right)^{5 / 3+\varepsilon}\right\} \leqslant y \leqslant x,
$$

where $u:=(\log x) / \log y$ and the implied constants depend on $a$ and $\varepsilon$.

Proof. Define

$$
f(d):= \begin{cases}d / \varphi(d) & \text { if }(a, d)=1 \\ 0 & \text { otherwise. }\end{cases}
$$

We shall apply Lemma 2.2 below to prove

$$
\sum_{\substack{d \leqslant x \\ P^{+}(d) \leqslant y}} f(d)=C_{a} \rho(u) x\left\{1+O_{a, \varepsilon}\left(\frac{\log (u+1)}{\log y}\right)\right\}
$$

uniformly for $(x, y) \in \mathcal{H}_{\varepsilon}$. To this end, it is sufficient to verify that the function $f$ verifies the conditions of Lemma 2.2.

With the help of the prime number theorem, it is easy to see that

$$
\begin{aligned}
\sum_{p \leqslant z} f(p) \log p & =\sum_{p \leqslant z, p \nmid a} \frac{p \log p}{p-1}=\sum_{p \leqslant z} \log p+O_{a}(\log z) \\
& =z+O_{a}\left(z \mathrm{e}^{-c(\log z)^{3 / 5}\left(\log _{2} z\right)^{-1 / 5}}\right)
\end{aligned}
$$

and

$$
\sum_{p} \sum_{\nu \geqslant 2} \frac{f\left(p^{\nu}\right)}{p^{\nu}} \leqslant \sum_{p, \nu \geqslant 2} \frac{1}{p^{\nu}\left(1-p^{-1}\right)} \leqslant \frac{2 \pi^{2}}{3} .
$$

This shows that $f$ satisfies the conditions (2.3) and (2.4) of Lemma 2.2. Thus the asymptotic formula (2.2) is a particular case of this general lemma.

Now the required result (2.1) follows from (2.2) by a simple partial summation.

Next we cite four lemmas, which are useful. The first one is a particular case of [14, Théorème 2.1] with $\kappa=1$ and $R(z)=\mathrm{e}^{c(\log z)^{3 / 5-\varepsilon}}$, which has been used in the proof of Lemma 2.1 above.

Lemma 2.2. Let $A>0, C>0, \eta \in\left(0, \frac{1}{2}\right)$ and $\varepsilon>0$. Suppose that arithmetic function $f: \mathbb{N} \rightarrow \mathbb{R}^{+}$verifies the following conditions

$$
\begin{gathered}
\left|\sum_{p \leqslant z} f(p) \log p-z\right| \leqslant C z \mathrm{e}^{-(\log z)^{3 / 5-\varepsilon}}, \\
\sum_{p} \sum_{\nu \geqslant 2} \frac{f\left(p^{\nu}\right)}{p^{(1-\eta) \nu}} \leqslant A .
\end{gathered}
$$


Then we have

$$
\sum_{\substack{n \leqslant x \\ P^{+}(n) \leqslant y}} f(n)=C(f) x \rho(u)\left\{1+O_{\varepsilon}\left(\frac{\log (u+1)}{\log y}\right)\right\}
$$

uniformly for $(x, y) \in\left(\mathcal{H}_{\varepsilon}\right)$, where

$$
C(f):=\prod_{p}(1-1 / p) \sum_{\nu \geqslant 0} f\left(p^{\nu}\right) / p^{\nu} .
$$

The second lemma is an elegant Brun-Titchmarsh inequality, due to MontgomeryVaughan [10].

Lemma 2.3. We have

$$
\sum_{\substack{x<p \leqslant x+y \\ p \equiv a(\bmod d)}} 1<\frac{2 y}{\varphi(d) \log (y / d)}
$$

uniformly for $1<d<y \leqslant x$ and $(a, d)=1$.

The third lemma is essentially due to Lachand and Tenenbaum [7]. *

Lemma 2.4. Let $\mu(n)$ be the Möbius function and let $P^{-}(n)$ be the largest prime factor with the convention $P^{-}(1)=\infty$. For any $\varepsilon>0$, we have

$$
\sum_{\substack{n \leqslant x \\ P^{-}(n)>y}} \frac{\mu(n)}{n}=\rho(u)+O_{\varepsilon}\left(\mathrm{e}^{-(\log y)^{3 / 5-\varepsilon}}\right)
$$

uniformly in

$$
x \geqslant 3 \quad \text { and } \quad \exp \left\{(\log x)^{2 / 5+\varepsilon}\right\} \leqslant y \leqslant x,
$$

where $u:=\log x / \log y$ and the implied constant depends on $\varepsilon$ only.

The last lemma is due to Iwaniec [6, Lemma 3] (see also [4, Lemme 4.1]).

Lemma 2.5. Let $D \geqslant 2$. There are two sequences $\left\{\lambda_{d}^{ \pm}\right\}_{d \geqslant 1}$, vanishing for $d>D$ or $\mu(d)=0$, verifying $\left|\lambda_{d}^{ \pm}\right| \leqslant 1$, such that

$$
\sum_{d \mid n} \lambda_{d}^{-} \leqslant \sum_{d \mid n} \mu(d) \leqslant \sum_{d \mid n} \lambda_{d}^{+} \quad(n \geqslant 1)
$$

and

$$
\begin{aligned}
& \sum_{d \mid P(z)} \lambda_{d}^{+} \frac{w(d)}{d} \leqslant \prod_{\substack{p \leqslant z \\
p \in \mathcal{P}}}\left(1-\frac{w(p)}{p}\right)\left\{F(s)+O\left(\frac{\mathrm{e}^{\sqrt{L}-s}}{\sqrt[3]{\log D}}\right)\right\} \\
& \sum_{d \mid P(z)} \lambda_{d}^{-} \frac{w(d)}{d} \geqslant \prod_{\substack{p \leqslant z \\
p \in \mathcal{P}}}\left(1-\frac{w(p)}{p}\right)\left\{f(s)+O\left(\frac{\mathrm{e}^{\sqrt{L}-s}}{\sqrt[3]{\log D}}\right)\right\}
\end{aligned}
$$

*In the original version of [7], there is a supplementary error term $O_{\varepsilon}\left(\exp \left\{-(\log y)^{3 / 5-\varepsilon}\right\}\right)$. Recently de la Bretèche \& Fiorilli [2] have succeeded to remove this superfluous error term. 
for any $z \in[2, D], s=(\log D) / \log z$, the set $\mathcal{P}$ of primes and the multiplicative function w satisfying

$$
\begin{aligned}
0 & <w(p)<p \quad(p \in \mathcal{P}), \\
\prod_{\substack{u<p \leqslant v \\
p \in \mathcal{P}}}\left(1-\frac{w(p)}{p}\right)^{-1} & \leqslant \frac{\log v}{\log u}\left(1+\frac{L}{\log u}\right) \quad(2 \leqslant u \leqslant v),
\end{aligned}
$$

where $P(z):=\prod_{p \leqslant z, p \in \mathcal{P}} p$, the implied $O$-constants are absolute and $F, f$ are defined by the continuous solutions to the system

$$
\begin{cases}s F(s)=2 \mathrm{e}^{\gamma} & (1 \leqslant s \leqslant 2), \\ s f(s)=0 & (0<s \leqslant 2), \\ (s F(s))^{\prime}=f(s-1) & (s>2) \\ (s f(s))^{\prime}=F(s-1) & (s>2) .\end{cases}
$$

Here $\gamma$ is the Euler constant.

\section{A VARIANT OF BombieRI-VinOGRADOV THEOREM}

The asymptotic behaviours of $\pi(x, y ; d, a)$ should be an interesting new subject in the prime number theory. An initial study on this counting function can be found in a recent work of Liu, Wu \& Xi [9]. The aim of this section is to establish (3.1) below by following their argument. We will see that it will play a key role in the proof of Theorem 1.

Proposition 3.1. Let $a \in \mathbb{Z}^{*}$ be a fixed non-zero integer, $\eta \in(0,1), \varepsilon>0$ and $A>0$. Assuming the Elliott-Halberstam conjecture $\mathrm{EH}[\eta]$, the following estimate

$$
\begin{aligned}
\sum_{\substack{d \leqslant \sqrt{x} \\
(a, d)=1 \\
P^{+}(d) \leqslant y}}\left|\pi(x, y ; d, a)-\frac{\pi(x)}{\varphi(d)} \rho\left(\frac{\log (x / d)}{\log y}\right)\right| & \ll_{a, A, \varepsilon} \frac{x}{(\log x)^{A}} \\
& +\pi(x)(\log y) \eta u \int_{0}^{u / 2} \rho(v) \mathrm{d} v
\end{aligned}
$$

holds uniformly for $\eta \in(0,1)$ and $(x, y) \in \mathcal{G}_{\varepsilon}$, where the implied constant depends on a, $A$ and $\varepsilon$ only.

Proof. Let $\mathfrak{P}:=\prod_{y<p \leqslant(x-a) / d} p$. The Möbius inversion formula allows us to write

$$
\begin{aligned}
\pi(x, y ; d, a) & =\sum_{\substack{p \leqslant x \\
p \equiv a(\bmod d)}} \sum_{\ell \mid(\mathfrak{P},(p-a) / d)} \mu(\ell)=\sum_{\substack{\ell \leqslant(x-a) / d \\
P^{-}(\ell)>y}} \mu(\ell) \pi(x ; d \ell, a) \\
& =\pi_{1}(x, y ; d, a)+\pi_{2}(x, y ; d, a),
\end{aligned}
$$


where

$$
\begin{aligned}
& \pi_{1}(x, y ; d, a):=\sum_{\substack{\ell \leqslant x^{1-\eta} / d \\
P^{-}(\ell)>y}} \mu(\ell) \pi(x ; d \ell, a), \\
& \pi_{2}(x, y ; d, a):=\sum_{\substack{x^{1-\eta} / d<\ell \leqslant(x-a) / d \\
P^{-}(\ell)>y}} \mu(\ell) \pi(x ; d \ell, a) .
\end{aligned}
$$

Firstly we treat $\pi_{1}(x, y ; d, a)$. We note, via writing $q=d \ell$,

$$
\sum_{\substack{d \leqslant \sqrt{x} \\(a, d)=1 \\ P^{+}(d) \leqslant y}}\left|\pi_{1}(x, y ; d, a)-\sum_{\substack{\ell \leqslant x^{1-\eta} / d \\ P^{-}(\ell)>y}} \frac{\mu(\ell)}{\varphi(d \ell)} \pi(x)\right| \leqslant \sum_{\substack{q \leqslant x^{1-\eta} \\(a, q)=1}} \tau(q)\left|\pi(x ; q, a)-\frac{\pi(x)}{\varphi(q)}\right| .
$$

Trivially, for all $q \leqslant x^{1-\eta}$, we have

$$
\pi(x ; q, a)+\frac{\pi(x)}{\varphi(q)} \ll \frac{x}{q} .
$$

Hence

$$
\sum_{\substack{q \leqslant x^{1-\eta} \\(a, q)=1}} \tau(q)^{2}\left|\pi(x ; q, a)-\frac{\pi(x)}{\varphi(q)}\right| \ll x \sum_{q \leqslant x^{1-\eta}} \frac{\tau(q)^{2}}{q} \ll x(\log x)^{4} .
$$

By (3.3) and the Cauchy-Schwarz inequality, this bound and the Elliott-Halberstam conjecture $\mathrm{EH}[\eta]$ with $2 A+4$ in place of $A$ allow us to derive that

$$
\sum_{\substack{d \leqslant \sqrt{x} \\(a, d)=1 \\ P^{+}(d) \leqslant y}}\left|\pi_{1}(x, y ; d, a)-\sum_{\substack{\ell \leqslant x^{1-\eta} / d \\ P^{-}(\ell)>y}} \frac{\mu(\ell)}{\varphi(d \ell)} \pi(x)\right| \ll_{a, A} \frac{x}{(\log x)^{A}}
$$

for all $x \geqslant 1$ and $y>1$.

Noticing that $(d, \ell)=1$ and using Lemma 2.4, we can write

$$
\begin{aligned}
\frac{\pi(x)}{\varphi(d)} \sum_{\substack{\ell \leqslant x^{1-\eta} / d \\
P^{-}(\ell)>y}} \frac{\mu(\ell)}{\varphi(\ell)} & =\frac{\pi(x)}{\varphi(d)} \sum_{\substack{\ell \leqslant x^{1-\eta} / d \\
P^{-}(\ell)>y}} \frac{\mu(\ell)}{\ell}\left\{1+O\left(\frac{1}{y}\right)\right\} \\
& =\frac{\pi(x)}{\varphi(d)} \rho\left(\frac{\log \left(x^{1-\eta} / d\right)}{\log y}\right)\left\{1+O_{\varepsilon}\left(\frac{\log (u+1)}{\log y}\right)\right\} .
\end{aligned}
$$

for all $(x, y) \in \mathcal{G}_{\varepsilon}$ and $d \leqslant \sqrt{x}$. On the other hand, for $d \leqslant \sqrt{x}$ we have ([13, Corollaries III.5.8.3-8.4])

$$
\begin{aligned}
\rho\left(\frac{\log (x / d)}{\log y}\right)-\rho\left(\frac{\log \left(x^{1-\eta} / d\right)}{\log y}\right) & \ll \eta u\left|\rho^{\prime}\left(\frac{\log (x / d)}{\log y}\right)\right| \\
& \ll \rho\left(\frac{\log (x / d)}{\log y}\right) \eta u \log (u+1) .
\end{aligned}
$$


JIE WU

Inserting it into the proceeding relation, it follows that

$$
\frac{\pi(x)}{\varphi(d)} \sum_{\substack{\ell \leqslant x^{1-\eta} / d \\ P^{-}(\ell)>y}} \frac{\mu(\ell)}{\varphi(\ell)}=\frac{\pi(x)}{\varphi(d)} \rho\left(\frac{\log (x / d)}{\log y}\right)\{1+O(\eta u \log (u+1))\}
$$

for all $(x, y) \in \mathcal{G}_{\varepsilon}$ and $d \leqslant \sqrt{x}$.

With the help of (2.1), a simple partial integration gives us

$$
\begin{aligned}
& \sum_{\substack{d \leqslant \sqrt{x} \\
(a, d)=1 \\
P^{+}(d) \leqslant y}} \frac{1}{\varphi(d)} \rho\left(\frac{\log (x / d)}{\log y}\right) \\
= & \pi(x) \int_{1-}^{\sqrt{x-a}} \rho\left(\frac{\log (x / t)}{\log y}\right) \mathrm{d}\left(\sum_{\substack{d \leqslant t,(a, d)=1 \\
P^{+}(d) \leqslant y}} \frac{1}{\varphi(d)}\right) \\
= & C_{a} \pi(x)(\log y) \int_{0}^{u / 2} \rho(v) \rho(u-v) \mathrm{d} v\left\{1+O_{a, \varepsilon}\left(\frac{\log (u+1)}{\log y}\right)\right\} \\
= & \frac{C_{a}}{2} \pi(x)(\log y)(\rho * \rho)(u)\left\{1+O_{a, \varepsilon}\left(\frac{\log (u+1)}{\log y}\right)\right\}
\end{aligned}
$$

for $(x, y) \in \mathcal{H}_{\varepsilon}$, we have used the trivial relation

$$
\int_{0}^{u / 2} \rho(v) \rho(u-v) \mathrm{d} v=\frac{1}{2} \int_{0}^{u} \rho(v) \rho(u-v) \mathrm{d} v=\frac{1}{2}(\rho * \rho)(u) .
$$

Combining (3.5) with (3.4) and using (3.6) to bound the contribution of the error term in $(3.5)$, we can find

$$
\begin{aligned}
\sum_{\substack{d \leqslant \sqrt{x} \\
(a, d)=1 \\
P^{+}(d) \leqslant y}}\left|\pi_{1}(x, y ; d, a)-\frac{\pi(x)}{\varphi(d)} \rho\left(\frac{\log (x / d)}{\log y}\right)\right| & \ll_{a, A, \varepsilon} \frac{x}{(\log x)^{A}} \\
& +\pi(x)(\log y)(\rho * \rho)(u) \eta u \log (u+1)
\end{aligned}
$$

for all $(x, y) \in \mathcal{G}_{\varepsilon}$ and $\eta \in(0,1)$, provided we assume $\mathrm{EH}[\eta]$.

We now turn to $\pi_{2}(x, y ; d, a)$. For $p-a=d \ell m$, we have $(a, m)=1$ and $m \leqslant x^{\eta}$ subject to the restrictions in $\pi_{2}(x, y ; d, a)$. We have trivially

$$
\left|\pi_{2}(x, y ; d, a)\right| \leqslant \sum_{\substack{x^{1-\eta} / d<\ell \leqslant(x-a) / d \\ P^{-}(\ell)>y}} \pi(x ; d \ell, a) \leqslant \sum_{\substack{m \leqslant x^{\eta} \\(a, m)=1}} \sum_{\substack{p \leqslant x, p \equiv a(\bmod d m) \\ P^{-}((p-a) / d m)>y}} 1 .
$$

We are now in a position to apply sifting arguments subject to the target sequence

$$
\mathcal{A}(x ; d m, a):=\{(p-a) / d m: p \leqslant x \text { and } p \equiv a(\bmod d m)\} .
$$

Trivially we have, with the notation $\mathfrak{P}_{2}(y):=\prod_{2<p \leqslant y} p$,

$$
\left|\pi_{2}(x, y ; d, a)\right| \leqslant \sum_{\substack{m \leqslant x^{\eta} \\(a, m)=1}} \sum_{\substack{n \in \mathcal{A}(x ; d m, a) \\\left(n, \mathfrak{P}_{2}(y)\right)=1}} 1
$$


Let $\left\{\lambda_{q}^{+}\right\}_{q \geqslant 1}$ be an upper bound sieve of level $Q$ as in Lemma 2.5, so that

$$
\begin{aligned}
\left|\pi_{2}(x, y ; d, a)\right| & \leqslant \sum_{\substack{m \leqslant x^{\eta} \\
(a, m)=1}} \sum_{n \in \mathcal{A}(x ; d m, a)} \sum_{q \mid\left(n, \mathfrak{P}_{2}(y)\right)} \lambda_{q}^{+} \\
& =\sum_{\substack{q \leqslant Q \\
q \mid \mathfrak{P}_{2}(y)}} \lambda_{q}^{+} \sum_{\substack{m \leqslant x^{\eta} \\
(a, m)=1}} \pi(x ; d m q ; a) .
\end{aligned}
$$

We may take $Q=x^{1 / 2-2 \eta}$ such that $\sqrt{x} Q x^{\eta}=x^{1-\eta}$. As before, we may approximate $\pi(x ; d m q ; a)$ on average over $d, m, q$ and apply the the Elliott-Halberstam conjecture $\mathrm{EH}[\eta]$. Similar to (3.3), we can prove

$$
\sum_{\substack{d \leqslant \sqrt{x} \\(a, d)=1 \\ P^{+}(d) \leqslant y}} \sum_{\substack{q \leqslant Q \\ q \mid \mathfrak{P}_{2}(y)}}\left|\lambda_{q}^{+}\right| \sum_{\substack{m \leqslant x^{\eta} \\(a, m)=1}}\left|\pi(x ; d m q ; a)-\frac{\pi(x)}{\varphi(d m q)}\right| \ll_{a, A} \frac{x}{(\log x)^{A}}
$$

for all $x \geqslant 2$ and $y>1$. It now follows that

$$
\sum_{\substack{d \leqslant \sqrt{x} \\(a, d)=1 \\ P^{+}(d) \leqslant y}}\left|\pi_{2}(x, y ; d, a)\right| \leqslant \pi(x) \sum_{\substack{d \leqslant \sqrt{x} \\(a, d)=1 \\ P^{+}(d) \leqslant y}} \sum_{\substack{m \leqslant x^{\eta} \\(a, m)=1}} \frac{1}{\varphi(d m)} \sum_{\substack{q \leqslant Q \\ q \mid \mathfrak{P}_{2}(y)}} \frac{\lambda_{q}^{+}}{\varphi(q)}+O_{a, A}\left(\frac{x}{(\log x)^{A}}\right) .
$$

From Lemma 2.5, Mertens' formula and the inequality $\varphi(d m) \geqslant \varphi(d) \varphi(m)$, we derive

$$
\begin{aligned}
\sum_{\substack{d \leqslant \sqrt{x} \\
(a, d)=1 \\
P^{+}(d) \leqslant y}}\left|\pi_{2}(x, y ; d, a)\right| & \ll_{a, A} \pi(x) \sum_{\substack{d \leqslant \sqrt{x} \\
P^{+}(d) \leqslant y}} \sum_{m \leqslant x^{\eta}} \frac{1}{\varphi(d m)} \prod_{2<p<y} \frac{p-2}{p-1}+\frac{x}{(\log x)^{A}} \\
& \ll_{a, A} \frac{\pi(x)}{\log y} \sum_{\substack{d \leqslant \sqrt{x} \\
P^{+}(d) \leqslant y}} \frac{1}{\varphi(d)} \sum_{m \leqslant x^{\eta}} \frac{1}{\varphi(m)}+\frac{x}{(\log x)^{A}} \\
& \ll_{a, A, \varepsilon} \pi(x)(\log y) \eta u \int_{0}^{u / 2} \rho(v) \mathrm{d} v+\frac{x}{(\log x)^{A}}
\end{aligned}
$$

for all $(x, y) \in \mathcal{H}_{\varepsilon}$ and $\eta \in(0,1)$. Now the required result follows from (3.7)-(3.8) and the trivial inequality

$$
(\rho * \rho)(u) \leqslant 2 \rho\left(\frac{u}{2}\right) \int_{0}^{u / 2} \rho(v) \mathrm{d} v \ll \frac{1}{\log (u+1)} \int_{0}^{u / 2} \rho(v) \mathrm{d} v .
$$

This completes the proof of Proposition 3.1.

\section{Proof of Theorem 1}

In view of the symmetry of divisors of integer $n$, we have

$$
\tau(n)=2 \sum_{\substack{d \mid n \\ d<\sqrt{n}}} 1+\delta_{\square}(n)
$$


with

$$
\delta_{\square}(n):= \begin{cases}1 & \text { if } n \text { is a perfect square } \\ 0 & \text { otherwise. }\end{cases}
$$

Thus we can write

$$
\begin{aligned}
\sum_{\substack{p \leqslant x \\
P^{+}(p-a) \leqslant y}} \tau(p-a) & =2 \sum_{\substack{d \leqslant(x-a)^{1 / 2} \\
(a, d)=1, P^{+}(d) \leqslant y}} \sum_{\substack{p \leqslant x \\
p \equiv a(\bmod d) \\
P((p-a) / d) \leqslant y}} 1+\sum_{\substack{p \leqslant x \\
P^{+}(p-a) \leqslant y}} \delta_{\square}(p-b) \\
& =2\left(\mathcal{M}+\mathcal{R}_{1}\right)+\mathcal{R}_{2},
\end{aligned}
$$

where

$$
\begin{aligned}
\mathcal{M} & :=\sum_{\substack{d \leqslant \sqrt{x-a} \\
(a, d)=1, P^{+}(d) \leqslant y}} \frac{\pi(x)}{\varphi(d)} \rho\left(\frac{\log (x / d)}{\log y}\right), \\
\mathcal{R}_{1} & :=\sum_{\substack{d \leqslant \sqrt{x-a} \\
(a, d)=1, P^{+}(d) \leqslant y}}\left(\pi(x, y ; d, a)-\frac{\pi(x)}{\varphi(d)} \rho\left(\frac{\log (x / d)}{\log y}\right)\right), \\
\mathcal{R}_{2} & :=\sum_{\substack{p \leqslant x \\
P^{+}(p-a) \leqslant y}} \delta_{\square}(p-b) .
\end{aligned}
$$

By noticing that $\delta_{\square}(p-b)=1 \Rightarrow p-b=n^{2}$, we have trivially

$$
\mathcal{R}_{2} \leqslant 2 \sqrt{x}
$$

uniformly for $x \geqslant 1$ and $y>1$.

According to Proposition 3.1, we have

$$
\mathcal{R}_{1} \ll_{a, A, \varepsilon} \frac{x}{(\log x)^{A}}+x \rho\left(\frac{u}{2}\right) \eta u
$$

uniformly for $(x, y) \in \mathcal{G}_{\varepsilon}$.

Finally according to (3.6), we have

$$
\mathcal{M}=\frac{C_{a}}{2} \pi(x)(\log y)(\rho * \rho)(u)\left\{1+O_{a, \varepsilon}\left(\frac{\log (u+1)}{\log y}\right)\right\}
$$

for $(x, y) \in \mathcal{H}_{\varepsilon}$.

Now inserting (4.4), (4.3) and (4.5) into (4.2) and using (3.9), we find that

$$
\begin{aligned}
\sum_{\substack{p \leqslant x \\
P^{+}(p-a) \leqslant y}} \tau(p-a)= & C_{a} \pi(x)(\log y)(\rho * \rho)(u)\left\{1+O_{a, \varepsilon}\left(\frac{\log (u+1)}{\log y}\right)\right\} \\
& +O_{a, A, \varepsilon}\left(\frac{x}{(\log x)^{A}}+\pi(x)(\log y) \eta u \int_{0}^{u / 2} \rho(v) \mathrm{d} v\right)
\end{aligned}
$$

uniformly for $(x, y) \in \mathcal{G}_{\varepsilon}$ and $\eta \in(0,1)$. Clearly (4.6) implies the required result (1.6) 


\section{Proof OF TheOREM 2}

5.1. Lower bound. Firstly we prove the lower bound in (1.6). Our principal tool is a mean value theorem of Bombieri-Vinogradov type in short intervals, due to $\mathrm{Wu}[17$, Théorme 1.2].

Lemma 5.1. Let $g(\ell)$ be an arithmetic function satisfying the condition

$$
\sum_{\ell \leqslant x}|g(\ell)|^{2} / \ell \ll(\log x)^{\lambda} \quad(x \geqslant 2)
$$

for some constant $\lambda>0$. Define

$$
H(z, h, d, a, \ell):=\sum_{\substack{\ell p \leqslant z+h \\ \ell p \equiv a(\bmod d)}} 1-\sum_{\substack{\ell p \leqslant z \\ \ell p \equiv a(\bmod d)}} 1-\frac{1}{\varphi(d)} \int_{z / \ell}^{(z+h) / \ell} \frac{\mathrm{d} t}{\log t} .
$$

Then for any $\frac{3}{5}<\theta \leqslant 1$ and $A>0$, there exists a constant $B=B(\theta, A)>0$ such that the inequality

$$
\sum_{d \leqslant D} \max _{(a, d)=1} \max _{h \leqslant y} \max _{x / 2<z \leqslant x}\left|\sum_{\ell \leqslant L,(\ell, d)=1} g(\ell) H(z, h, d, a, \ell)\right| \ll_{\theta, \varepsilon} \frac{y}{(\log x)^{A}}
$$

holds for $x \geqslant 3, D:=x^{\theta-1 / 2} /(\log x)^{B}$ and $L=x^{(5 \theta-3) / 2}$.

In view of (4.1), we have trivially

$$
\tau(n)=\sum_{d \mid n} 1 \geqslant 2 \sum_{\substack{d \mid n \\ d<n^{\theta-1 / 2}}} 1
$$

Thus we can write

$$
\begin{aligned}
& \sum_{x<p \leqslant x+y} \tau(p-a) \geqslant 2 \sum_{\substack { x<p \leqslant x+y \\
\begin{subarray}{c}{d<(p-a)^{\theta-1 / 2} \\
d \mid(p-a){ x < p \leqslant x + y \\
\begin{subarray} { c } { d < ( p - a ) ^ { \theta - 1 / 2 } \\
d | ( p - a ) } }\end{subarray}} 1 \\
& \geqslant 2 \sum_{\substack{d \leqslant x^{\theta-1 / 2}(\log x)^{B} \\
(a, d)=1}} \sum_{\substack{x<p \leqslant x+y \\
p \equiv a(\bmod d)}} 1 \\
&=2(\mathscr{M}+\mathscr{R}),
\end{aligned}
$$

where

$$
\begin{aligned}
& \mathscr{M}:=\sum_{\substack{d \leqslant x^{\theta-1 / 2}(\log x)^{B} \\
(a, d)=1}} \frac{1}{\varphi(d)} \int_{x}^{x+y} \frac{\mathrm{d} t}{\log t},
\end{aligned}
$$

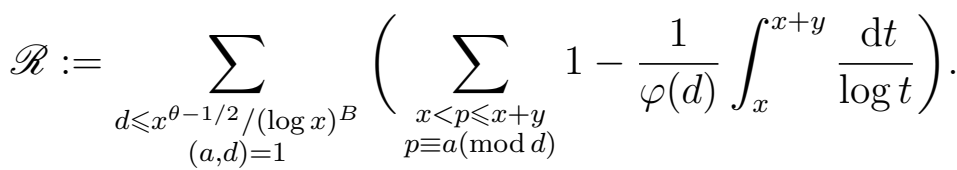

In Lemma 5.1, taking

$$
g(\ell)= \begin{cases}1 & \text { if } \ell=1 \\ 0 & \text { otherwise }\end{cases}
$$


we can obtain

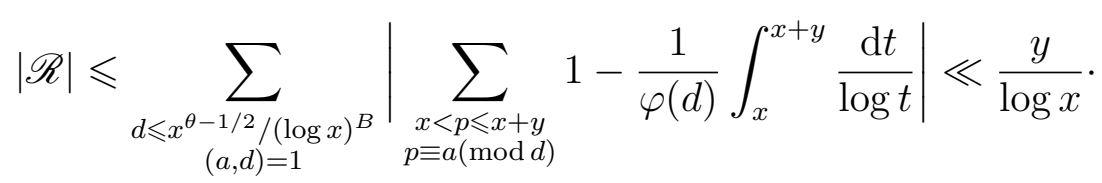

By using (2.1) with $y=x$, it follows that

$$
\begin{aligned}
\mathscr{M} & =\left\{\left(\theta-\frac{1}{2}\right) C_{a} \log x+O\left(\log _{2} x\right)\right\} \int_{x}^{x+y} \frac{\mathrm{d} t}{\log t} \\
& =\left(\theta-\frac{1}{2}\right) C_{a} y\left\{1+O\left(\frac{\log _{2} x}{\log x}\right)\right\} .
\end{aligned}
$$

Inserting (5.7) and (5.6) into (5.3), we obtain the lower bound in (1.6).

5.2. Upper bound. Now we prove the bound bound in (1.6). By (4.1), we write

$$
\begin{aligned}
\sum_{x<p \leqslant x+y} \tau(p-a) & \leqslant 2 \sum_{x<p \leqslant x+y} \sum_{\substack{d \mid(p-a) \\
d<\sqrt{p-a}}} 1+2 \sqrt{x} \\
& \leqslant 2(\mathscr{M}+\mathscr{R}+\mathscr{S}+\sqrt{x})
\end{aligned}
$$

where $\mathscr{M}$ and $\mathscr{R}$ are defined as in (5.4)-(5.5) and

$$
\mathscr{S}:=\sum_{x^{\theta-1 / 2} /(\log x)^{B}<d \leqslant \sqrt{3 x}} \sum_{\substack{x<p \leqslant x+y \\ p \equiv a(\bmod d)}} 1 .
$$

In the preceding subsection, we have proved that

$$
\mathscr{M}+\mathscr{R}=\left(\theta-\frac{1}{2}\right) C_{a} y\left\{1+O\left(\frac{\log _{2} x}{\log x}\right)\right\} .
$$

On the other hand, by the Brun-Titchmarsh inequality (2.6), it follows that

$$
\begin{aligned}
\mathscr{S} & <\sum_{x^{\theta-1 / 2} /(\log x)^{B}<d \leqslant \sqrt{3 x}} \frac{2 y}{\varphi(d) \log (y / d)} \\
& =\frac{2 y}{\log y} \sum_{x^{\theta-1 / 2 /(\log x)^{B}<d \leqslant \sqrt{3 x}}} \frac{1}{\varphi(d)(1-(\log d) / \log y)} .
\end{aligned}
$$

In view of (2.1) with $y=x$, a simple partial integration allows us to deduce that

$$
\begin{aligned}
\mathscr{S} & =\frac{2 y}{\log y} \int_{x^{\theta-1 / 2} /(\log x)^{B}-}^{\sqrt{3 x}} \frac{1}{1-(\log t) / \log y} \mathrm{~d} \sum_{d \leqslant t} \frac{1}{\varphi(d)} \\
& =\left\{1+O\left(\frac{\log _{2} x}{\log x}\right)\right\} 2 C_{a} y \int_{1-1 /(2 \theta)}^{1 /(2 \theta)} \frac{1}{1-v} \mathrm{~d} v \\
& \leqslant\left\{1+O\left(\frac{\log _{2} x}{\log x}\right)\right\} 2 \log (2 \theta-1)^{-1} C_{a} y .
\end{aligned}
$$

New the upper bound in (1.6) follows from (5.9), (5.10) and (5.8). This completes the proof of Theorem 2. 
Acknowledgement. This work is partially supported by NSFC (Grant $\mathrm{N}^{\circ}$ 11771121).

\section{REFERENCES}

[1] E. Bombieri, J. B. Friedlander \& H. Iwaniec, Primes in arithmetic progressions to large moduli, Acta Math. 156 (1986), 203-251.

[2] R. de la Bretèche \& D. Fiorilli, Entiers friables dans des progressions arithmétiques de grand module, arXiv:1506.03268v1 [math.NT] 10 June 2015.

[3] É. Fouvry, Sur le problme des diviseurs de Titchmarsh, J. Reine Angew. Math. 37 (1985), 51-76.

[4] É. Fouvry \& G. Tenenbaum, Répartition statistique des entiers sans grand facteur premier dans les progressions arithmétiques, Proc. London Math. Soc. 72 (1996) 481514.

[5] A. Granville, Smooth numbers: computational number theory and beyond, Algorithmic number theory: lattices, number fields, curves and cryptography, 44 (2008), 267-323.

[6] H. Iwaniec, A new form of the error term in the linear sieve, Acta Arith. 37 (1980), 307-320.

[7] A. Lachand \& G. Tenenbaum, Note sur les valeurs moyennes criblées de certaines fonctions arithmétiques, Quart. J. Math. 66 (2015), no. 1, 245-250.

[8] Yu. V. Linnik, The dispersion method in binary additive problems, (Leningrad 1961), Transl. Math. Monographs, Vol.4, Amer. Math. Soc., Providence, R.I., 1963.

[9] J.-Y. Liu, J. Wu \& P. Xi, Primes in arithmetic progressions with friable indices, Preprint, 2017.

[10] H. L. Montgomery \& R. C. Vaughan, On the large sieve, Mathematika, 20 (1973), 119-134.

[11] P. Pollack, A Titchmarsh divisor problem for elliptic curves, Math. Proc. Camb. Phil. Soc. 160 (2016), no. 1, 167-189.

[12] C. Pomerance, Popular values of Euler's function, Mathematika 27 (1980), 84-89.

[13] G. Tenenbaum, Introduction to analytic and probabilistic number theory, Translated from the second French edition (1995) by C. B. Thomas, Cambridge Studies in Advanced Mathematics 46, Cambridge University Press, Cambridge, 1995. xvi+448 pp.

[14] G. Tenenbaum \& J. Wu, Moyennes de certaines fonctions multiplicatives sur les entiers friables, J. Reine Angew. Math. 564 (2003), 119-167.

[15] E. C. Titchmarsh, A divisor problem, Rend. Circ. Mat. Palermo 54 (1930), 414-429, with correction, 478-479.

[16] Z. Wang, Autour des plus grands facteurs premiers d'entiers consécutifs voisins d'un entier criblé, Mathematika, to appear.

[17] J. Wu, Théorèmes généralisés de Bombieri-Vinogradov dans les petits intervalles, Quart. J. Math. Oxford (2), 44 (1993), 109-128.

[18] P. Xi, A quadratic analogue of Titchmarsh divisor problem, J. Number Theory, 184 (2018), $192-205$.

Jie Wu, Department of Mathematics, Southwest University, 2 Tiansheng Road, Beibei, 400715 Chongqing, China

Current address: CNRS UMR 8050, Laboratoire d'Analyse et de Mathématiques Appliquées, Université Paris-Est Créteil, 61 avenue du Général de Gaulle, 94010 Créteil Cedex, France

E-mail address: jie.wu@math.cnrs.fr 